\title{
Introduction to the Minitrack on Service Analytics
}

\author{
Hansjörg Fromm, Niklas Kühl, Gerhard Satzger \\ Karlsruhe Institute of Technology (KIT) \\ Karlsruhe Service Research Institute (KSRI) \\ hansjoerg.fromm@kit.edu \\ niklas.kuehl@kit.edu \\ gerhard.satzger@kit.edu
}

The Minitrack on Service Analytics is part of the Decision Analytics, Mobile Services and Service Science Track of the 54th Annual Hawaii International Conference on System Sciences (HICSS-54) on January 5-8, 2021.

Service Analytics describe all processes of capturing, processing, and analyzing data taken from a service system - in order to improve, extend, and personalize the service provided and to create new value for both the provider and the customer.

The modern view on services focuses on the cocreation of value between providers and customersleveraging knowledge, skills, and resources of both partners from an overall system point of view. In most service systems, however, the service providers have no access to data related to the service usage by their customers. On the other hand, an increasing volume of data will be collected either by the users/customers themselves (e.g. through wearables or mobile/smart phones) or by technologies like smart metering in energy services, telematics in automotive and mobility services, RFID in logistics, machine condition sensors in manufacturing, or data capture solutions in healthcare.

Research topics addressed in this year's minitrack and in future installations of the minitrack include the applicability of basic and advanced analytics to different service systems, the state-of-the-art of service analytics methodologies and tool-support, and the investigation of benefits resulting from the application of service analytics.

This minitrack will serve as a forum for researchers and practitioners to share progress in the study of these and related themes. Submissions on, but not limited to, the following topics are encouraged:

-Web Usage Mining and Web Personalization

- Data Mining / Machine Learning applied to Services

- Recommender Systems for Services

\author{
Thomas Setzer \\ Catholic University of Eichstätt-Ingolstadt (KU) \\ Information Systems and Business Informatics \\ thomas.setzer@ku.edu
}

- Social Network Analytics applied to Services

-Privacy Issues resulting from Service Analytics

- Fraud Analytics for Service Systems

-Analysis and Prediction of User Behavior in

Mobile Phone Systems

-Analysis and Prediction of Driver Behavior in

Traffic Situations

- Analysis and Exploitation of Floating Car Data

- Electricity Consumption Analysis using Smart

Meter Data

- Analytics for Healthcare Services

-Analysis and Prediction of IT Service

Demand Patterns

- Analysis of Service Problem Reports

- Industrial Service Analytics and Optimization

-Sports Analytics

The following research papers have been accepted for publication in the HICSS proceedings and will be presented at this year's Service Analytics Minitrack:

-Towards a Machine Learning-based Decision Support System for Dispatching Helicopters in New Zealand, by Tim Rädsch, Dave Richards, and Melanie Reuter-Oppermann

- Upgrading Products based on Existing Dominant Competitors, by Syauki Thamrin, Arbee L.P. Chen, and Heri Wijayanto

-Usage Space Sampling for Fringe Customer Identification, by Jan Thiele, Kunxiong Ling, and Thomas Setzer 